\title{
Frequency mixing in accelerator based sources and application to tunable seeded free-electron lasers
}

\author{
C. Evain, E. Roussel, C. Szwaj, and S. Bielawski \\ Laboratoire de Physique des Lasers, Atomes et Molécules, UMR CNRS 8523, \\ Université des Sciences et Technologies de Lille, F-59655 Villeneuve d'Ascq Cedex, France
}

(Received 6 December 2013; published 29 December 2014)

\begin{abstract}
In this article we address the problem of tunability of seeded free-electron lasers (FELs), working typically in the domain of the vacuum ultraviolet. The seeding of FELs with an external laser permits us to obtain FEL pulses with both good longitudinal coherence and good stability properties (contrary to an amplification starting from noise). However, with a fixed external laser wavelength, only amplification at harmonics of this wavelength is possible. If full tunability is wanted, it is necessary to have a tunable external source; but this type of source has much less power, hence it is much harder to reach high harmonics numbers. Here we propose the so-called frequency mixing scheme (from its analogy with the similar process in nonlinear optics), based on two laser/electron interactions. Numerical and analytic studies show that it permits to increase significantly the tunability at short wavelengths using a low-energy tunable source. Numerical simulations are made with the parameters of FERMI-FEL 1, the first seeded FEL for user operation.
\end{abstract}

DOI: 10.1103/PhysRevSTAB.17.120706

PACS numbers: 41.60.Cr, 42.25.Kb, 42.65.Ky

\section{INTRODUCTION}

Free-electron lasers (FELs) are powerful light sources, particularly used in the domain of the vacuum ultraviolet (VUV) and of the X-rays [1]. The last generation of FELs are the single-pass FELs, where a relativistic electronbunch goes through a series of undulators (composed of magnets creating a periodic transverse magnetic field) [2]. In these undulators, the interaction of the electrons with their own synchrotron radiation leads to a light amplification, which can be of several orders of magnitude, until wavelengths as short as about one angstrom [2-5].

In the traditional operation, the so-called SASE mode (self-amplification of stimulated emission) [2], the amplification starts from spontaneous emission, which decreases considerably the longitudinal coherence and the reproducibility of the emitted pulses [6].

Hence, the longitudinal coherence properties and the stability are important issues of single pass FELs and several strategies are developed to improve them. One strategy is the so-called self-seeding, where an optical monochromator [7] or a crystal [8] is used to filter the emission at an intermediate point in the amplification process. It permits us to increase significantly the coherence properties (in the X-ray domain) [8-10].

*clement.evain@phlam.univ-lille1.fr

Published by the American Physical Society under the terms of the Creative Commons Attribution 3.0 License. Further distribution of this work must maintain attribution to the author(s) and the published article's title, journal citation, and DOI.
Another strategy consists in seeding the FEL with external laser pulse(s), in order to transfer both the good coherence properties and the stability of these external laser pulses to the FEL pulses. Two methods are mainly applied, working in the domain of the VUV and of the soft X-rays. One method consists of seeding directly the FEL with a source from harmonic from gas $[11,12]$, but their low energy at short wavelengths is a limitation, and the tunability is not full (since this source is not fully tunable). The other methods are the harmonic schemes, based on the creation of micro-structures in the longitudinal profile of the bunch at harmonics of the external laser wavelength, before the amplification process. Traditionally, the laser seeds used are obtained from a Ti:Sa oscillator, coupled with an amplifier. The simplest harmonic scheme is the HGHG scheme (high gain harmonic generation scheme) [13,14], based on one laser/electron interaction, and permits us to reach harmonic numbers of the order of ten [5]. In this scheme, if the laser seed has a fixed wavelength, only wavelength at harmonic of this seed can be amplified and thus the tunability is not full. To increase the tunability, it is possible to use a tunable source [typically from an oscillator parametric amplifier (OPA)] [15,16] but either only low frequencies are accessible (since to reach high harmonic numbers, high energy laser pulse is needed), or an important laser system (with a lower repetition rate) is needed [17].

Here we propose a new scheme, called frequency mixing scheme, from its analogy with the nonlinear optical process [18]. This scheme is based on two laser-electron interactions (like the recent schemes as the EEHG scheme $[19,20]$ or the difference mixing scheme [21]) and permits us to create new wavelengths in the short wavelength 
domain (typically in the frequency range accessible by HGHG). Associated to an OPA source, it permits us to increase significantly the tunability of seeded FELs at high harmonic numbers. This study is done numerically and analytically using for illustration the parameters of FERMIFEL1, the first seeded FEL for users [5]. A comparison of the efficiency between the HGHG scheme using a lowenergy tunable source, and the proposed method (using also a low-energy tunable source) shows a gain of orders of magnitudes, when "high" harmonic numbers are targeted.

\section{PRINCIPLE}

The general layout of this so-called frequency mixing scheme is composed of two laser/electron interactions, occurring either in one undulator [Fig. 1(a)] or in two undulators [Fig. 1(b)], provided an energy exchange arises at both wavelengths. Then the electron-bunch goes through a dispersive section (typically composed of three bending magnets), used to transfer the energy modulation to the longitudinal profile. At this step, the induced microstructures can be used to start the light amplification in tuned undulators.

At first, numerical simulations are made in the configuration of one undulator [Fig. 1(a)] tuned at $800 \mathrm{~nm}$. The first laser wavelength $\lambda_{1}$ is fixed at $260 \mathrm{~nm}$ and the second laser wavelength $\lambda_{2}$ is fixed at $800 \mathrm{~nm}$. For the accelerator parameters, these simulations are done in the case of FERMI-FEL1 (see the caption of Fig. 2 for the parameter value).

Figure 2 shows the electron-bunch longitudinal characteristics at each step of the process, using a macro-particle code, where each of the $N_{\mathrm{mp}}$ macro-particles is characterized by its longitudinal position $z$ (in meters) and its relative energy $p=\left(E-E_{0}\right) / \sigma_{E}$ (with $E$ the electron energy, $E_{0}$ the average beam energy and $\sigma_{E}$ the bunch energy-spread).

Before the laser/electron interactions, the electron bunch distribution $f(z, p)$ (in unit of the number of macroparticles per unit of length and of energy) is assumed to be Gaussian in energy and uniform in position [Fig. 2(a)].

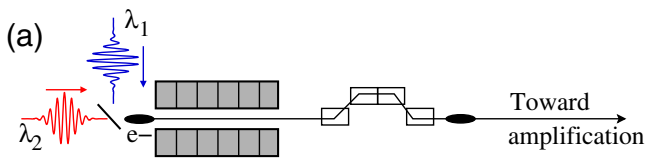

(b)

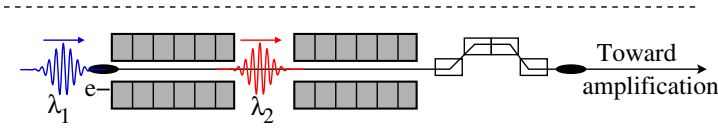

FIG. 1. Layout of the frequency mixing process. First step: interaction between an electron-bunch and two external laser pulses of wavelength $\lambda_{1}$ and $\lambda_{2}$, in (a) one or (b) two undulators. Second step: passage of the electrons through a dispersive section (composed of three bending magnets). The induced microstructures can then be used to start light amplification in undulators.
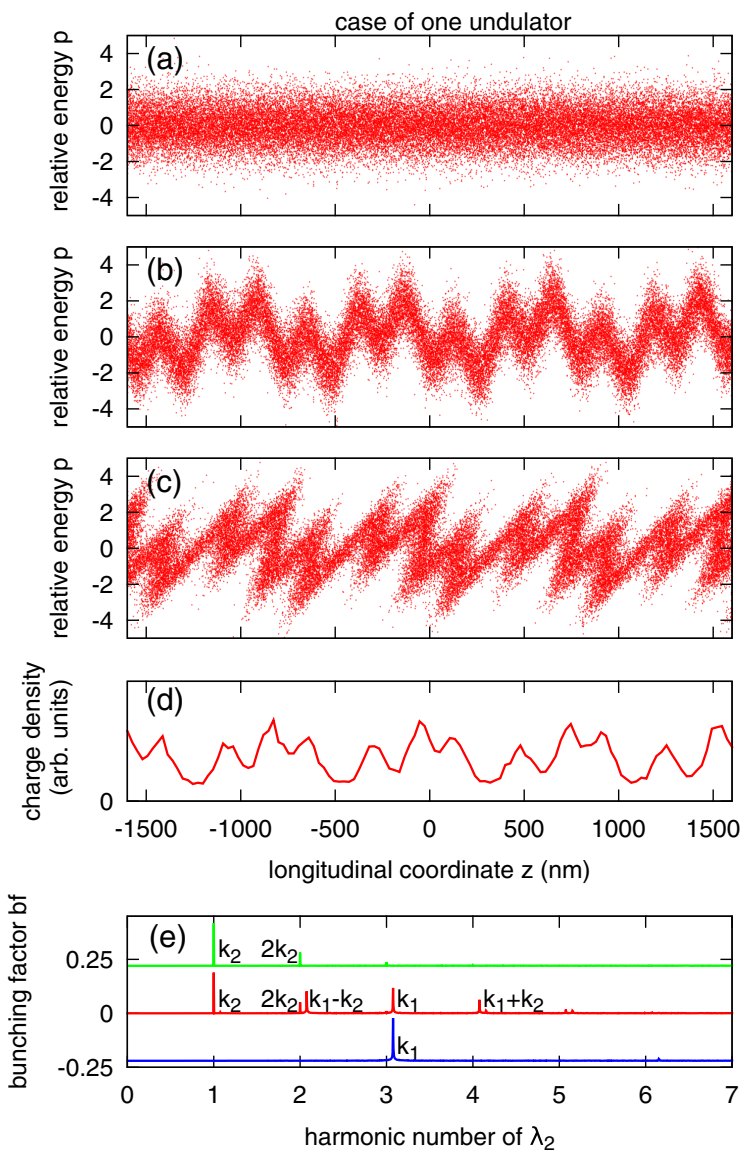

FIG. 2. Electron bunch longitudinal characteristics at each step of the frequency mixing process (case of one undulator). Phasespace of the electron-bunch, (a) initially, (b) after the interaction with the 2 external lasers, and (c) after the dispersive section. Associated (d) charge density $\rho(z, t)$ and (e) bunching factor $b f$ (red line). Blue and green lines: bunching factor obtained with only one laser (blue line: $E_{2}=0$, green line: $E_{1}=0$. For clarity these bunching factors are shifted by +0.22 and -0.22 , respectively). Parameters: $E_{0}=1.2 \mathrm{GeV}, \sigma_{E}=150 \mathrm{keV}, N_{1}=30$, $\lambda_{w 1}=100 \mathrm{~mm}, \quad \lambda_{m 1}=800 \mathrm{~nm}, \quad \lambda_{1}=260 \mathrm{~nm}, \quad \lambda_{2}=800 \mathrm{~nm}$, $E_{1}=1 \mathrm{GV} / \mathrm{m}, E_{2}=0.18 \mathrm{GV} / \mathrm{m}, w_{1}=500 \mu \mathrm{m}, w_{2}=870 \mu \mathrm{m}$, $R_{56}=4.554 \times 10^{-4} \mathrm{~m}, N_{\mathrm{mp}}=2 \times 10^{6}$. The bunching factors are calculated over 200 periods of $\lambda_{m 1}$ and each period is discretized in $N_{\text {bin }}=30$ cases.

The laser/electron interactions are simulated by integrating the equations (1)-(6) [22] over the length of the undulator(s) (with a different normalization compared to [22] and using an Euler method).

$$
\begin{gathered}
\frac{d p}{d z}=\frac{e}{\sigma_{E}} E_{x} \beta_{x} \\
E_{x}=\frac{E_{1}}{\sqrt{1+\left(z / z_{r 1}\right)^{2}}} \sin \left[k_{1}(z-c t)+\psi_{1}\right] \\
+\frac{E_{2}}{\sqrt{1+\left(z / z_{r 2}\right)^{2}}} \sin \left[k_{2}(z-c t)+\psi_{2}\right]
\end{gathered}
$$




$$
\begin{gathered}
t=\frac{z}{c \times \beta_{z}} \\
\beta_{x}=-\frac{K_{1} m_{0} c^{2}}{E_{0}+p \times \sigma_{E}} \sin \left(\frac{2 \pi}{\lambda_{w 1}} z\right) \\
\beta_{z}=\sqrt{1-\frac{\left(m_{0} c^{2}\right)^{2}}{\left(E_{0}+p \times \sigma_{E}\right)^{2}}-\beta_{x}^{2}} \\
K_{1}=\sqrt{2\left(E_{0} / m_{0} c^{2}\right)^{2} \frac{\lambda_{m 1}}{\lambda_{w 1}}-1}
\end{gathered}
$$

These equations describe the interaction of one electron (of charge $e$ and of mass $m_{0}$ ) with an electric field $E_{x}$ composed of two wave numbers $k_{1}=2 \pi / \lambda_{1}$ and $k_{2}=2 \pi / \lambda_{2}$. This interaction occurs in one undulator (composed of $N_{1}$ periods of length $\lambda_{w 1}$, with a resonant wavelength $\lambda_{m 1}$ ). To simulate the interactions in two undulators, these equations are integrated twice, the first time with $E_{2}=0$ and the second time with $E_{1}=0$, with $E_{1}$ and $E_{2}$ the electric field amplitude associated to $k_{1}$ and $k_{2}$, respectively (the parameters of the second undulator are noted $N_{2}, \lambda_{m 2}, \lambda_{w 2}$ ). The laser pulses are also defined by their Rayleigh length $z_{r i}$ (or their waist $w_{i}=\sqrt{\lambda_{i} z_{r i} / \pi}$, localized at the middle of the undulator) and the Gouy Phase shift $\psi_{i}=-\arctan \left(z / z_{r i}\right)$ (with $i=1$ and $i=2$ associated to $k_{1}$ and $k_{2}$, respectively). $c$ is the light velocity. To facilitate the interpretation, the lasers are taken uniform in the $z$ direction. It is also assumed that the energy modulation is uniform along the transverse coordinates, i.e., it is assumed that the laser beam size is larger that the transverse electron bunch dimensions.

The laser-induced energy modulation amplitudes are noted $A_{1}$ and $A_{2}$ and are in unit of the energy spread $\sigma_{E}$. In this example, the value of $E_{1}$ and $E_{2}$ are chosen to get an energy modulation amplitude (when only one laser pulse is used) of $A_{1} \simeq A_{2} \simeq 1$ [Fig. 2(b)].

After the interaction with the lasers, the electron bunch goes through the dispersive section. In the approximation of a linear transport [23], it induces a change of longitudinal position $\delta z$ proportional to $p$ and to the transport matrix element $R_{56}: \delta z=p \times R_{56} \frac{\sigma_{E}}{E_{0}}$ [Fig. 2(c)].

The micro-structures in the longitudinal profile $\rho(z)$ bunch are observable making the projection of the phase-space $f(z, p)$ along the energy axis $[\rho(z)=$ $\left.\int_{-\infty}^{+\infty} f(z, p) d p\right]$ [Fig. 2(d)]. This projection is a nonlinear process, and looking at its frequency components (more precisely, looking at the bunching factor $b f(k)=$ $\left.\frac{1}{N_{m p}}\left|\int_{-\infty}^{+\infty} \rho(z) e^{-i k z} d z\right|\right)$, we can observe the effects of the frequency mixing process: the presence of frequency components at some combinations of the two laser wave numbers: $k=k_{1}-k_{2}$ and $k=k_{1}+k_{2}$ [cf. Fig. 2(e)]. In this example, the value of $R_{56}$ is chosen to obtain a significant bunching factor at the wave number $k_{1}+k_{2}$ and $k_{1}-k_{2}$, but there are also micro-structures at the fundamentals $\left(\lambda_{1}, \lambda_{2}\right)$ and harmonics (mainly at $\left.\lambda_{2} / 2\right)$ of the laser wavelengths. This electron bunch can then initialize the amplification process in undulators tuned at the desired frequency. Only the components in the FEL bandwidth will be amplified.

\section{TUNABILITY WITH A TUNABLE SEED-THE CASE OF TWO UNDULATORS}

In a second time, to study the tunability of this scheme, numerical simulations are made using a tunable laser seed. We first consider the case of two undulators. The first laser wavelength is fixed at $260 \mathrm{~nm}$, with an energy pulse of $130 \mu \mathrm{J}$ (obtainable from a $100 \mathrm{fs}$ FWHM, $1 \mathrm{~mJ}$ pulse at $800 \mathrm{~nm}$, coupled to a third harmonic process in a nonlinear crystal), and the second laser wavelength is taken tunable, from $500 \mathrm{~nm}$ to $1000 \mathrm{~nm}$, with a lower energy pulse of $10 \mu \mathrm{J}$, also obtainable from a $1 \mathrm{~mJ}$ pulse at $800 \mathrm{~nm}$ (coupled to an OPA system). To optimize the energy modulation process [22] at $260 \mathrm{~nm}$, the first undulator is tuned at $267 \mathrm{~nm}$, and for simplicity the other one tuned at the tunable wavelength $\lambda_{2}$ (between 500 and $900 \mathrm{~nm}$ ). the dispersive section strength $R_{56}$ is kept fixed and chosen to be near the maximum bunching factor at the wave number $k=7 k_{1}+k_{2}$.

Figure 3(a) shows the bunching factor (in grey scale) in function of the micro-structure wavelength (horizontal axis) and of the tunable wavelength (vertical axis). Two plots of the bunching factor are also presented in Fig. 3(b), at the wavelength $\lambda_{2}=800 \mathrm{~nm}$ and $\lambda_{2}=700 \mathrm{~nm}$. For

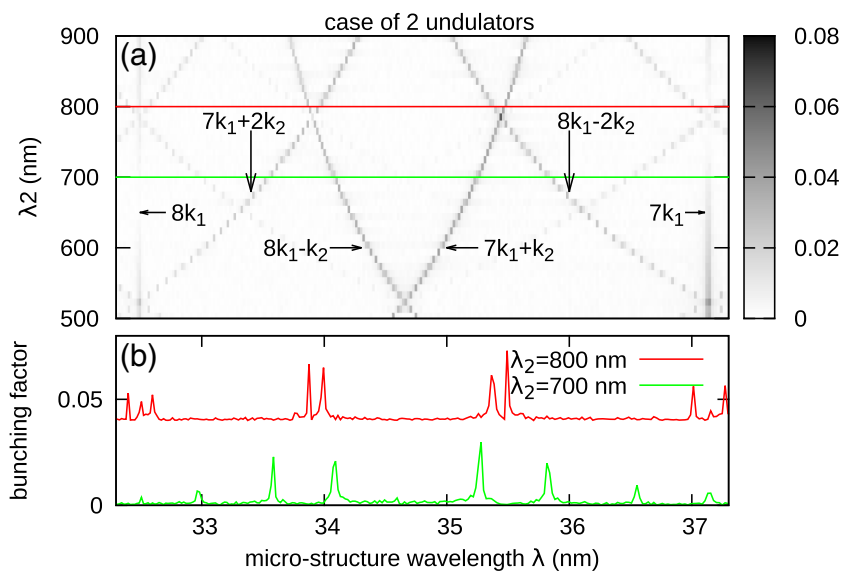

FIG. 3. (a) Bunching factor $b f(\lambda)$ versus $\lambda_{2}$ in the case of 2 undulators, with $\lambda_{m 1}=267 \mathrm{~nm}, \lambda_{m 2}=\lambda_{2}$, and $R_{56}=$ $8.35 \times 10^{-5} \mathrm{~m}$. (b) Detail for (red) $\lambda_{2}=800 \mathrm{~nm}$ and (green) $\lambda_{2}=700 \mathrm{~nm}$ (for figure clarity, the red line is vertically shifted by 0.04). Same other parameters that in Fig. 2 except $E_{1}=1.5 \mathrm{GV} / \mathrm{m}, \quad E_{2}=0.25 \mathrm{GV} / \mathrm{m}, \quad N_{2}=N_{1}, \quad \lambda_{\omega 2}=\lambda_{\omega 1}$, $N_{\text {bin }}=40$. These parameters permit us to have an energy modulation of about $A_{1} \simeq 5$ and $A_{2} \simeq 1\left(A_{2}=1.4\right.$ at $800 \mathrm{~nm}$ and $A_{2}=0.82$ at $500 \mathrm{~nm}$ ). 
the figure clarity, we choose to focus only on the micro-structure wavelengths between the 7th and the 8th harmonic of the first laser (i.e., between $37.143 \mathrm{~nm}$ and $32.5 \mathrm{~nm})$.

We observe [Fig. 3(a,b)] the presence of significant micro-bunching at several frequency combinations; mainly at $k=7 k_{1}+k_{2}, k=8 k_{1}-k_{2}, k=7 k_{1}+2 k_{2}$, and $k=$ $8 k_{1}-2 k_{2}$. Thus, full tunability can be achieve between $7 k_{1}$ and $8 k_{1}$, since in this wavelength domain, there is always a combination of $m$ and $n$ where the bunching factor is higher than the bunching factor associated to the shot-noise. Similar results can be obtained in other frequency domains (at least for longer wavelength, for example between $k_{1}=6$ and $k_{1}=7$ ), only the $R_{56}$ value has to be optimized.

\section{TUNABILITY WITH A TUNABLE SEED-The CASE OF ONE UNDULATOR}

In the case of one undulator, the parameters are taken to be identical as that for the two undulator case, except that the undulator is tuned at the fixed frequency $\lambda_{m 1}=790 \mathrm{~nm}$. In Fig. 4(a,b), we observe significant micro-bunching mainly at $k=7 k_{1}+k_{2}$ and $k=8 k_{1}-k_{2}$. The limitation to obtain stronger micro-structures over all the frequencies comes from the undulator resonance bandwidth. As the undulator resonant wavelength $\lambda_{m 1}$ is kept fixed, the energy modulation $A_{2}$ is small far from the resonance, as well as the bunching factor. However, it still permits us to have some tunability. For example, for the combination $k=7 k_{1}+k_{2}$, a bunching factor higher than $1 \%$ exists between about $35.813 \mathrm{~nm}$ and $35.135 \mathrm{~nm}$ (associated to $\lambda_{2}=1000 \mathrm{~nm}$ and $\lambda_{2}=650 \mathrm{~nm}$, respectively), that is about $15 \%$ of the frequency domain between $7 k_{1}$ and $8 k_{1}$. A similar ratio can also be obtained between other harmonics of $\lambda_{1}$.

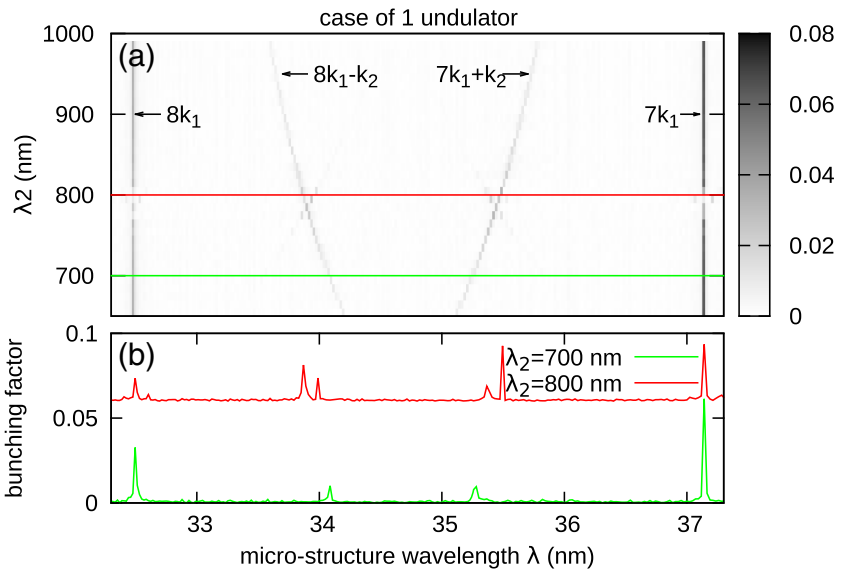

FIG. 4. (a) Bunching factor $b f(\lambda)$ versus $\lambda_{2}$ in the case of 1 undulator. (b) Detail for (red) $\lambda_{2}=800 \mathrm{~nm}$ and (green) $\lambda_{2}=$ $700 \mathrm{~nm}$ (for figure clarity, the red line is vertically shifted by 0.06). Other parameters are the same as in Fig. 3 except $\lambda_{m 1}=790 \mathrm{~nm}$ and $R_{56}=10.3 \times 10^{-5} \mathrm{~m}$.

\section{POWER LAW-ANALOGY WITH CLASSICAL OPTICS}

Then, it is possible to have an analytic estimation of the bunching factor $b_{n, m}$ at the wave number $k=n k_{1}+m k_{2}$ (with $n$ and $m$ integer) in function of the parameters [cf. Eq. (7)], using the analytic expression obtained in the frame of the difference mixing scheme [21] or in the frame of the EEHG scheme [20].

$$
\begin{aligned}
b_{n, m}= & \mid e^{-\frac{1}{2}\left((K m+n) B_{2}\right]^{2}} \times J_{m}\left[-(K m+n) A_{2} B_{2}\right] \\
& \times J_{n}\left[-(K m+n) A_{1} B_{2}\right] \mid
\end{aligned}
$$

with $K=\frac{k_{2}}{k_{1}}, B_{2}=R_{56} k_{1} \frac{\sigma_{E}}{E_{0}}$ and $J_{i}$ the Bessel function of order $i$. Equation (7) is obtained in the approximation of infinitely long bunch and laser pulses, and of sinusoidal energy modulations (induced by the lasers).

Figure 5 shows the bunching factor [calculated with (7)] versus $A_{2}$, in the configuration of Fig. 3 (with $A_{1}=5$ and $K=3.07$, case of $\lambda_{1}=260 \mathrm{~nm}$ and $\lambda_{2}=800 \mathrm{~nm}$ ), and at the wave number $k=7 k_{1}+k_{2}$ and $k=7 k_{1}+2 k_{2}$. For small values of $A_{2}$ (until about $A_{2} \simeq 1$ ), the bunching factor scales as the power law $A_{2}^{m}$, since $J_{m}(z)$ can be approximated by $J_{m}(z) \simeq \frac{1}{\Gamma(m+1)}\left(\frac{z}{2}\right)^{m}$ (with $\Gamma$ the gamma function). For higher values of $A_{2}$, a saturation process occurs. When no saturation occurs, neither from $A_{1}$ nor from $A_{2}$, the bunching factor is proportional to $E_{1}^{n} \times E_{2}^{m}$ ( $A_{i}$ is proportional to $E_{i}$ [22], with $\left.i=1,2\right)$. Since without amplification process, the coherent power is proportional to $|b f|^{2}$ [24], we find here the same power law that in classical nonlinear frequency mixing process, where the power emitted at the wave number $n k_{1}+m k_{2}$ is equal to $\chi^{2(n+m)} E_{1}^{2 n} \times E_{2}^{2 m}$, with $\chi^{(n+m)}$ the susceptibility of order $n+m$ of the nonlinear material [18].
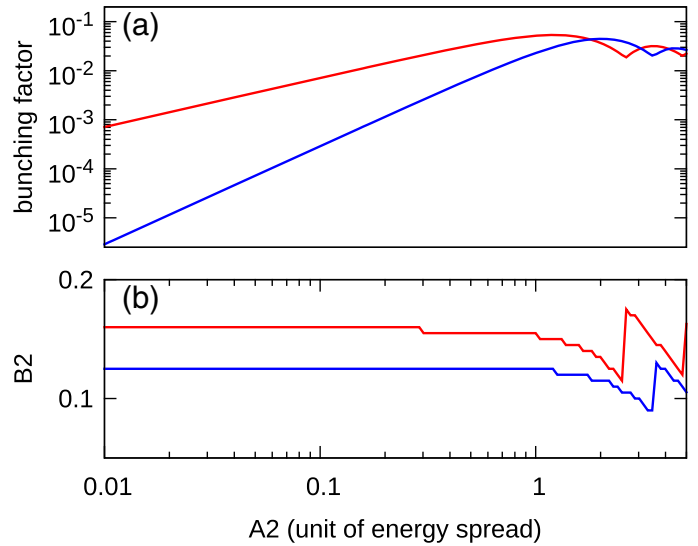

FIG. 5. (a) Bunching factor versus $A_{2}$, from Eq. (7), with $K=3.07, A_{1}=5$ and (red line) $n=7, m=1$ and (blue line) $n=7, m=2$. (b) Associated value of $B_{2}$ maximizing the bunching factor. 
This scaling law permits us also to understand how this scheme can be used to get tunability at "short" wavelengths (in the range accessible by HGHG), using a "low" energy tunable laser system. A first laser with a strong power and a fixed wavelength is used to reach short wavelengths ("high" value of $n$ ), whereas a second laser, tunable but with a lower energy (i.e., permitting us to reach only a small value of $m$ ) is used to achieve the tunability.

\section{COMPARISON WITH HGHG WITH A LOW ENERGY OPA SOURCE}

Then we have compared the efficiency of this scheme (using either 1 or 2 undulators) with the efficiency of the HGHG scheme at a high harmonic number, when a relative small power is available (10 $\mu \mathrm{J}$ for the tunable source and $130 \mu \mathrm{J}$ at $260 \mathrm{~nm}$ ). We focus on four micro-structure wavelengths around $35 \mathrm{~nm}: 35.5 \mathrm{~nm}, 34.6 \mathrm{~nm}, 34 \mathrm{~nm}$, and $32.4 \mathrm{~nm}$. For the HGHG scheme, these wavelengths are obtained from the 7 th harmonic $(m=7)$ of the laser seed (cf. Table I). For the frequency mixing scheme, these wavelengths are associated to the 7th harmonic of the $\lambda_{1}=260 \mathrm{~nm}(n=7)$ and to the first $(m=1)$ or second $(m=2)$ harmonic of $\lambda_{2}=800 \mathrm{~nm}$ or $\lambda_{2}=500 \mathrm{~nm}$ (cf. Table I). To estimate the bunching factor, we use the formula (7). For each case, the value of $A_{2}$ and $A_{1}$ are obtained using the numerical code presented in Sec. II, and the value of $B_{2}$ is optimized to get the maximum bunching factor (cf. Table I).

In these conditions, the bunching factor obtained with the HGHG scheme is quite small $\left(b f<3 \times 10^{-4}\right)$. The bunching factor obtained with the frequency mixing scheme is higher about two orders of magnitude, except in the case of one undulator, when $\lambda_{2}$ is too far from the undulator resonant wavelength $\lambda_{m 1}$. In these conditions, the bunching factor becomes null.

In the case where a stronger laser is available, the HGHG process will become also very efficient. But in these conditions, the frequency mixing scheme can still be used to reach higher frequencies, since a higher power at $260 \mathrm{~nm}$ will be available and so higher harmonic numbers can be reached. Nevertheless some other effects also have to be taken into account, as the FEL efficiency decrease due to an energy spread increase. As a first indication, the comparison with the Pierce parameter $\rho[5,25]$ gives a maximum energy modulation of about $10 \sigma_{E}$ to still have a FEL amplification.

\section{COMPARISON WITH HGHG ON THE INDUCED ENERGY-SPREAD}

Since the energy-spread is an important parameter for the FEL efficiency, we finally compare the minimum energyspread needed by the frequency mixing scheme and by the HGHG scheme to reach a given bunching factor. This comparison is done in the function of the wanted bunching factor (cf. Fig. 6) at the wavelength of $35.5 \mathrm{~nm}$ (see Table I for the configuration of the two schemes), using Eq. (7). For the HGHG scheme, a scan of the value of $A_{1}$ and of $B_{2}$ is done, and the minimum value of $A_{1}$ is kept when the wanted bunching factor is reached. For the frequency mixing scheme, a scan of $A_{1}, A_{2}$, and $B_{2}$ is done, and the minimum of $\left(A_{1}+A_{2}\right)$ is kept when the wanted bunching factor is reached. The results are presented in Fig. 6.

The HGHG scheme induces less energy spread than the frequency mixing scheme, by a factor of about $40 \%$.

TABLE I. Bunching factor at $35.5 \mathrm{~nm}, 34.6 \mathrm{~nm}, 34 \mathrm{~nm}$, and $32.4 \mathrm{~nm}$ obtained with the HGHG scheme and with the frequency mixing scheme (with 1 or 2 undulators), using a moderate laser source $\left(10 \mu \mathrm{J}\right.$ at $\lambda_{2}$ and $130 \mu \mathrm{J}$ at $\left.\lambda_{1}=260 \mathrm{~nm}\right)$. The energy modulation amplitude is obtained from numerical simulations (cf. Sec. II) with the same parameters as in Fig. 3 unless otherwise mentioned (the values of $\lambda_{m 2}$ has to be optimized to obtain the maximum value of $A_{2}$ ). $A_{1}=5$ for the frequency mixing scheme and $A_{1}=0$ for the HGHG scheme. For each case, $B_{2}$ is optimized to get the maximum bunching factor.

\begin{tabular}{|c|c|c|c|c|}
\hline Micro-structure wavelength (nm) & Laser \& undulator parameters & $A_{2}$ & $B_{2}$ & Bunching factor $b f$ \\
\hline \multicolumn{5}{|l|}{ HGHG scheme } \\
\hline $35.5(m=7, n=0)$ & $\lambda_{2}=248.5 \mathrm{~nm}, \lambda_{m 2}=255 \mathrm{~nm}, w_{2}=500 \mu \mathrm{m}$ & $A_{2}=1.3$ & 0.12 & $1.8 \times 10^{-4}$ \\
\hline $34.6(m=7)$ & $\lambda_{2}=242.2 \mathrm{~nm}, \lambda_{m 2}=250 \mathrm{~nm}, w_{2}=500 \mu \mathrm{m}$ & $A_{2}=1.4$ & 0.12 & $2.9 \times 10^{-4}$ \\
\hline $34.0(m=7)$ & $\lambda_{2}=238.0 \mathrm{~nm}, \lambda_{m 2}=245 \mathrm{~nm}, w_{2}=500 \mu \mathrm{m}$ & $A_{2}=1.4$ & 0.12 & $2.9 \times 10^{-4}$ \\
\hline $32.4(m=7)$ & $\lambda_{2}=226.8 \mathrm{~nm}, \lambda_{m 2}=235 \mathrm{~nm}, w_{2}=500 \mu \mathrm{m}$ & $A_{2}=1.4$ & 0.12 & $2.9 \times 10^{-4}$ \\
\hline \multicolumn{5}{|c|}{ Frequency mixing scheme - 2 Undulators } \\
\hline $35.5(n=7, m=1)$ & $\lambda_{2}=800 \mathrm{~nm}, \lambda_{m 2}=805 \mathrm{~nm}, w_{2}=870 \mu \mathrm{m}$ & 1.5 & 0.145 & $5.0 \times 10^{-2}$ \\
\hline $34.6(n=7, m=1)$ & $\lambda_{2}=500 \mathrm{~nm}, \lambda_{m 2}=510 \mathrm{~nm}, w_{2}=870 \mu \mathrm{m}$ & 1.15 & 0.17 & $5.3 \times 10^{-2}$ \\
\hline $34.0(n=7, m=2)$ & $\lambda_{2}=800 \mathrm{~nm}, \lambda_{m 2}=805 \mathrm{~nm}, w_{2}=870 \mu \mathrm{m}$ & 1.5 & 0.12 & $3.8 \times 10^{-2}$ \\
\hline $32.4(n=7, m=2)$ & $\lambda_{2}=500 \mathrm{~nm}, \lambda_{m 2}=510 \mathrm{~nm}, w_{2}=870 \mu \mathrm{m}$ & 1.15 & 0.15 & $2.8 \times 10^{-2}$ \\
\hline \multicolumn{5}{|c|}{ Frequency mixing scheme - 1 Undulator } \\
\hline $35.5(n=7, m=1)$ & $\lambda_{2}=800 \mathrm{~nm}, \lambda_{m 1}=795 \mathrm{~nm}, w_{2}=870 \mu \mathrm{m}$ & 1.1 & 0.15 & $5.3 \times 10^{-2}$ \\
\hline $34.6(n=7, m=1)$ & $\lambda_{2}=500 \mathrm{~nm}, \lambda_{m 1}=795 \mathrm{~nm}, w_{2}=870 \mu \mathrm{m}$ & 0 & & 0 \\
\hline $34.0(n=7, m=1)$ & $\lambda_{2}=800 \mathrm{~nm}, \lambda_{m 1}=795 \mathrm{~nm}, w_{2}=870 \mu \mathrm{m}$ & 1.1 & 0.12 & $2.6 \times 10^{-2}$ \\
\hline $32.4(n=7, m=1)$ & $\lambda_{2}=500 \mathrm{~nm}, \lambda_{m 1}=795 \mathrm{~nm}, w_{2}=870 \mu \mathrm{m}$ & 0 & & 0 \\
\hline
\end{tabular}



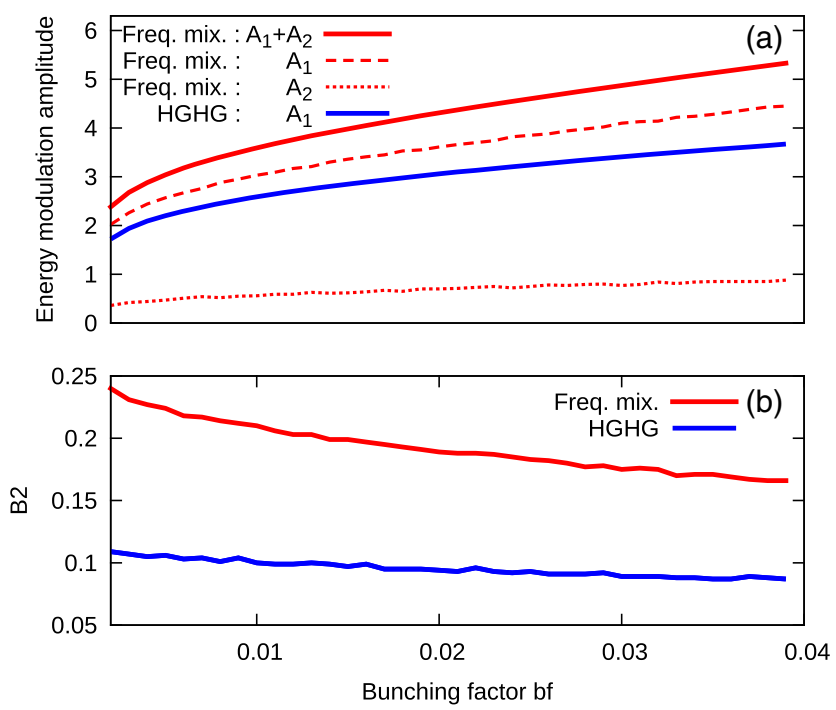

FIG. 6. (a) Minimum energy-spread modulation amplitude in function of the wanted bunching factor, for the HGHG scheme (blue line), and the frequency mixing scheme (Red lines, plain line: $A_{1}+A_{2}$ and dashed lines: details of $A_{1}$ and $A_{2}$ ). (b) Associated value of $B 2$ maximizing the bunching factor. Comparison done at $35.5 \mathrm{~nm}$ using Eq. (7).

We also observe that the second energy modulation $A_{2}$ needed with the frequency mixing scheme is weaker than the other energy modulations.

\section{CONCLUSION}

This numerical and analytic study shows that the frequency mixing scheme in relativistic electron bunches (which have similar scaling laws as the frequency mixing process in nonlinear optics) can be used to seed FELs and get significant tunability at short wavelengths (typically in the VUV domain). One of the two external seeds needs to be tunable, but contrary to a direct seeding, only a moderate power is sufficient, since the short wavelengths are reached thanks to the second seed (which has a fixed wavelength and thus can easily have more energy). Compared to the HGHG scheme this scheme requests an increase of energy spread by a factor of about $40 \%$.

This analytic and numerical study is focused on the amplitude of the induced micro-structures; besides experimental investigation, an important next step will be to perform free-electron laser simulations to get the temporal and spectral distributions of the output FEL pulses.

\section{ACKNOWLEDGMENTS}

This work was supported by the French "Agence Nationale de la Recherche" (ANR) (Blanc 2010-042301) and the "Laboratoire d'Excellence CEMPI."

[1] E. Saldin, E. Schneidmiller, and M. Yurkov, The Physics of Free Electron Lasers (Springer, New York, 2010).

[2] B. W. J. McNeil and N. R. Thompson, Nat. Photonics 4, 814 (2010).

[3] P. Emma et al., Nat. Photonics 4, 641 (2010).

[4] T. Ishikawa et al., Nat. Photonics 6, 540 (2012).

[5] E. Allaria et al., Nat. Photonics 6, 699 (2012).

[6] E. L. Saldin, E. A. Schneidmiller, and M. V. Yurkov, New J. Phys. 12, 035010 (2010).

[7] J. Feldhaus, E. Saldin, J. Schneider, E. Schneidmiller, and M. Yurkov, Opt. Commun. 140, 341 (1997).

[8] G. Geloni, V. Kocharyan, and E. Saldin, J. Mod. Opt. 58, 1391 (2011).

[9] J. Amann et al., Nat. Photonics 6, 693 (2012).

[10] A. Marinelli, C. Pellegrini, L. Giannessi, and S. Reiche, Phys. Rev. ST Accel. Beams 13, 070701 (2010).

[11] G. Lambert et al., Nat. Phys. 4, 296 (2008).

[12] S. Ackermann et al., Phys. Rev. Lett. 111, 114801 (2013).

[13] L. H. Yu, Phys. Rev. A 44, 5178 (1991).

[14] L.-H. Yu et al., Science 289, 932 (2000).

[15] E. Allaria, New J. Phys. 14, 113009 (2012).

[16] B. Liu et al., Phys. Rev. ST Accel. Beams 16, 020704 (2013).

[17] M. B. Danailov, A. Demidovich, R. Ivanov, I. Nikolov, P. Sigalotti, and P. Cinquegrana, Proceedings of FEL11, Shangai, China (2011), p. 183, https://accelconf.web .cern.ch/accelconf/FEL2011/papers/tuoc4.pdf.

[18] Y. R. Shen, The Principles of Nonlinear Optics (Wiley Classics Library, New York, 2002).

[19] G. Stupakov, Phys. Rev. Lett. 102, 074801 (2009).

[20] D. Xiang and G. Stupakov, Phys. Rev. ST Accel. Beams 12, 030702 (2009).

[21] D. Xiang and G. Stupakov, Phys. Rev. ST Accel. Beams 12, 080701 (2009).

[22] A. Zholents and K. Holldack, Proceedings of FEL2006, BESSY, Berlin, Germany (2006), p. 725, https://accelconf .web.cern.ch/accelconf/f06/PAPERS/THPPH059.PDF.

[23] A. W. Chao, Physics of Collective Beam Instabilities in High Energy accelerators (John Wiley \& Sons, New York, 1993).

[24] J. S. Nodvick and D. S. Saxon, Phys. Rev. 96, 180 (1954).

[25] K.-J. Kim, Phys. Rev. Lett. 57, 1871 (1986). 